\title{
Two novel $V C P$ missense variants identified in Japanese patients with multisystem proteinopathy
}

\author{
Michio Inoue ${ }^{1,2}$, Aritoshi lida ${ }^{3}$, Shinichiro Hayashi ${ }^{1}$, Madoka Mori-Yoshimura ${ }^{4}$, Atsushi Nagaoka ${ }^{5}$, Shunsuke Yoshimura ${ }^{5}$, \\ Hirokazu Shiraishi ${ }^{5}$, Akira Tsujino ${ }^{5}$, Yuji Takahashi ${ }^{4}$, Ikuya Nonaka', Yukiko K. Hayashi ${ }^{6}$, Satoru Noguchi ${ }^{1}$ and \\ Ichizo Nishino (1) ${ }^{1,3}$
}

\begin{abstract}
VCP mutations were first associated with inclusion body myopathy with Paget's disease of bone and frontotemporal dementia (IBMPFD) but was later associated with amyotrophic lateral sclerosis and Charcot-Marie-Tooth disease. Now, a new name, "multisystem proteinopathy (MSP)", is proposed for this condition. VCP encodes valosin-containing protein, which is involved in protein degradation in the ubiquitin proteasome system. We report here two MSP patients with two novel heterozygous missense variants in VCP: c.259G>T (p.Val87Phe) and c.376A>G (p.lle126Val).
\end{abstract}

\section{Data report}

$V C P$ mutations have been associated with (1) myopathy pathologically characterized by the presence of rimmed vacuoles, which is often called inclusion body myopathy (IBM), (2) Paget's bone disease, or (3) frontotemporal dementia, or in some cases, all of these conditions. Therefore, the disease was collectively termed inclusion body myopathy with Paget disease of bone and frontotemporal dementia (IBMPFD) ${ }^{1}$. In the largest cohort studies of patients with VCP disease, myopathy was seen in $89 \%$ of patients, Paget's disease of bone was seen in $43 \%$, and dementia was seen in $30 \%^{2}$. The phenotype has now been expanded to amyotrophic lateral sclerosis (ALS) and Charcot-Marie-Tooth disease. VCP mutations account for $2 \%$ of familial ALS cases ${ }^{3}$. The prevalence of the disease is not known in Japan. However, as a referral center for muscle disease diagnosis, we have diagnosed 22 patients (20 families) with $V C P$ mutations so far,

\footnotetext{
Correspondence: Ichizo Nishino (nishino@ncnp.go.jp)

'Department of Neuromuscular Research, National Institute of Neuroscience, National Center of Neurology and Psychiatry, Tokyo 187-8551, Japan

${ }^{2}$ Integrated Graduate School of Medicine, Engineering, and Agricultural

Science, University of Yamanashi, Yamanashi 409-3898, Japan

Full list of author information is available at the end of the article
}

including previous reports ${ }^{4,5}$. Due to the expanded phenotype, the term multisystem proteinopathy (MSP) is now proposed for a group of disorders that includes those associated with $V C P$ mutations ${ }^{6}$. So far, 54 different mutations have been reported (Human Gene Mutation Database); however, a genotype-phenotype correlation has not been clearly established for most of these mutations, and the incidence, prevalence, penetrance, and history of VCP diseases have not been clarified. VCP is an essential AAA + ATPase that is conserved in eukaryotes ${ }^{7}$. It is involved in major proteolytic pathways in cellular homeostasis, including membrane fusion, DNA damage repair, cell cycle and protein degradation. VCP forms a homo-hexamer with each monomer, comprising an $\mathrm{N}$ terminal domain (NTD) and a pair of ATP domains, D1 and D2. A series of missense mutations, which mostly occur at the NTD-D1 interface, dominant-negatively cause specific malfunctions of protein homeostasis linked to degenerative disorders ${ }^{8}$. Thus, patient muscle pathology commonly shows cytoplasmic and nuclear protein accumulation in muscle fibers, together with rimmed vacuoles. Previously, we have reported seven Asian patients with VCP myopathy with/without bone and brain phenotypes ${ }^{4}$. The skeletal muscle pathologies 

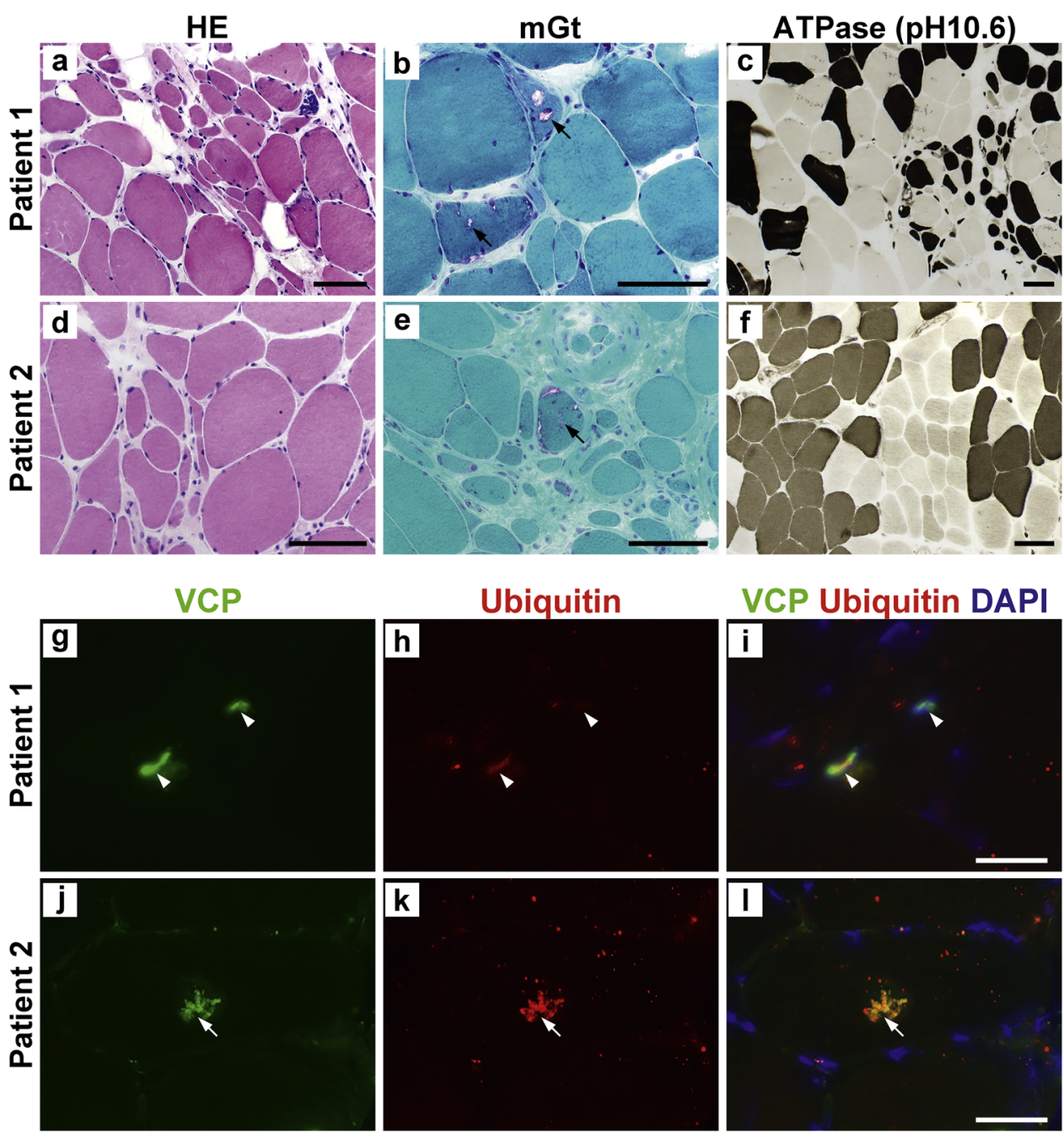

Fig. 1 a-f: Histological analysis of the muscle biopsies from patients. a, d: Hematoxylin and eosin (HE), (b, e): modified Gomori trichrome (mGt), (c, f): ATPase (pH10.6). A group of atrophic fibers was observed in both patients $(\mathbf{a}, \mathbf{c}, \mathbf{d}, \mathbf{f})$. mGt staining revealed the presence of rimmed vacuoles (b, e, black arrows). Bar: 50 um. g-l: Immunostaining for valosin-containing protein (VCP: $\mathbf{g}, \mathbf{i}, \mathbf{j}, \mathbf{I})$ and ubiquitin (h, i, $\mathbf{k}, \mathbf{l})$. VCP positive inclusions were co-stained with ubiquitin in both the nucleus (white arrowheads, DAPI positive) and the cytoplasm (white arrows, DAPI negative). Bar: $50 \mu m$

indicated mixed neurogenic and myogenic changes, fibers with rimmed vacuoles, and the presence of cytoplasmic and nuclear inclusions. In this study, we report two novel heterozygous missense variants (c.259G $>\mathrm{T}$ (p.Val87Phe); c.376A $>$ G (p.Ile126Val)) in $V C P$ in two Japanese patients with MSP.

National Center of Neurology and Psychiatry (NCNP) is a referral center for neuromuscular diseases in Japan. Since 1978, we have performed pathological diagnoses on more than 18,000 muscle biopsies. For cases with undiagnosed hereditary muscle disease, we now perform mutation screening using an Ion PGM sequencer (Thermo Fisher Scientific, MA, USA) in combination with targeted gene panels that we recently developed to cover 187 known causative genes for hereditary muscle diseases in four panels: muscular dystrophy, congenital myopathy, metabolic myopathy, and myopathy with protein aggregations/rimmed vacuoles (myofibrillar myopathy [MFM] panel; Supplementary Table S1) ${ }^{9}$.

Patient 1: The patient was a 73-year-old man with no family history of neuromuscular disease. He showed progressive muscle weakness and atrophy with involvement of the axial and proximal muscles. His symptoms started at the age of 71 as difficulty standing up. He also presented with psychological and cognitive impairment, including hallucinations and depression. His Mini-Mental State Examination and Frontal Assessment Battery scores were 26 and 11, respectively, suggesting mild cognitive dysfunction. He experienced difficulty in urination, suggesting autonomic dysfunction. He showed diminished deep tendon reflex, no pathologic reflex and no fasciculation. His serum CK levels were normal. There was no 
a

\section{Patient 1}

c.376A>G (p.lle126Val)
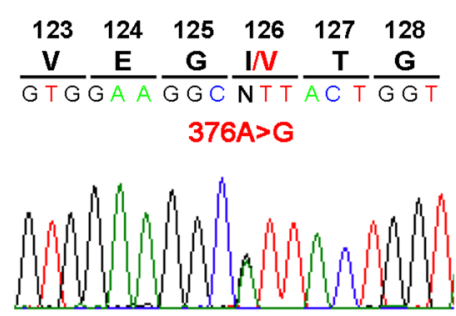

Patient 2

c.259G>T (p.Val87Phe)
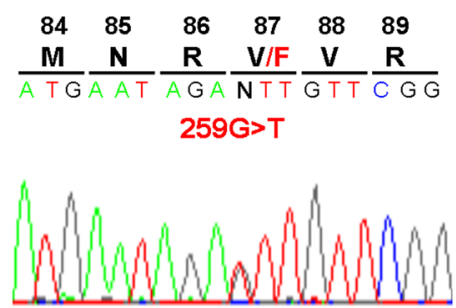

b

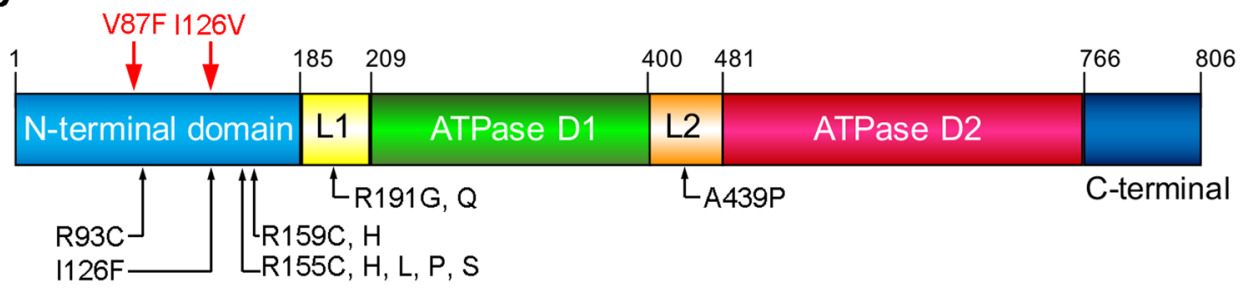

C

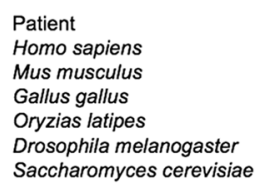

Saccharomyces cerevisiae

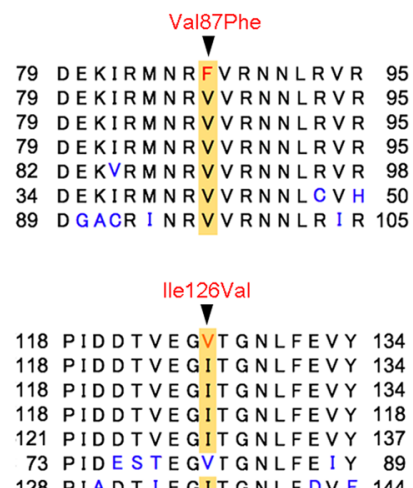

Val87Phe

79 DEKIRMNRFVRNNLRVR 95 DEKIRMNRVVRNNLRVR 95 DEKIRMNRVVRNNLRVR 95 DEKIRMNRVVRNNLRVR 95 DEKVRMNRVVRNNLRVR 98 4 DEKIRMNRVVRNNLCVH 50 DGACR I NRVVRNNLRIR 105

Patient

Homo sapiens

Mus musculus

Gallus gallus

Oryzias latipes

hila melanogaster

73 PIDESTEGVTGNLFEIY 89

128 PIADTIEGITGNLFDVF 144 d

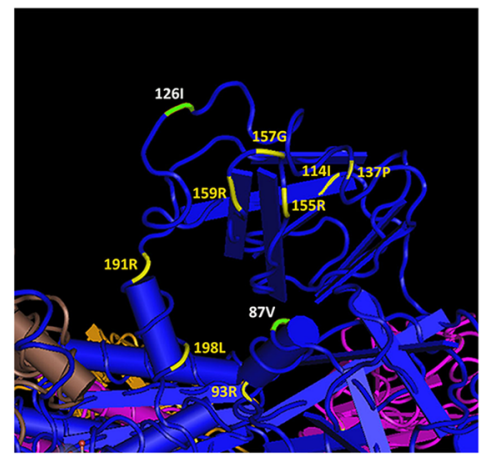

Fig. 2 Results of valosin-containing protein (VCP) mutation screening. a Two novel heterozygous substitutions were identified (c.376A>G and C. $259 \mathrm{G}>$ T). $\mathbf{b}$ Schematic functional domains and mutations of VCP. The locations of substitutions in this study are denoted by red arrows. Black arrows indicate the locations of previously identified mutations with inclusion body myopathy (IBM). c Multiple alignment of the VCP amino acid sequence in different species. The substitution sites in this study (Val87 and Ile126) are highly evolutionarily conserved. $\mathbf{d}$ The crystal structure of VCP protein showing the locations of the mutated residues detected in IBM. The crystal structure was obtained from the Molecular Modeling Database (MMDB ID: 82738). Our substitution sites (Val87 and Ile126) and those of previously reported mutations are indicated in white and yellow, respectively

sign of bone involvement, e.g., elevated serum alkaline phosphatase (ALP) levels. A nerve conduction study showed reduced compound muscle action potential without evidence of slowed conduction velocities. Needle electromyography (EMG) showed decreased recruitment, suggesting neurogenic change. Brain single photon emission computed tomography showed diminished blood flow to the occipital lobe. MIBG myocardial scintigraphy revealed reduced uptake. Muscle biopsy showed mixed changes of myopathy and neuropathy (Fig. 1a-c). Scattered fibers with rimmed vacuoles and a few fibers with cytoplasmic bodies were seen (Fig. 1b,g-i). In addition, groups of small angular fibers and fiber type groupings were seen (Fig. 1a,c).
Patient 2: The patient was a 65-year-old man with no family history of neuromuscular disease. In his late 40s, his initial symptom was difficulty raising his arms. Then, he developed progressive, mildly asymmetric upper limbonset muscle weakness with facial involvement and scapular winging with sensory involvement. At 59 years old, he was unable to stand up without support. Over time, his respiratory function declined, and respiratory failure appeared at age 65 years, leading to death at age 66. His serum CK levels ranged from 246 to $669 \mathrm{U} / \mathrm{L}$. No cognitive impairment was noted. He had no sign of bone involvement either in plain spinal X-ray or plain CT of the whole body. ALP testing data were not available. Needle EMG showed early recruitment, suggesting myogenic 
change. Muscle biopsy showed mixed changes, indicating myopathy and neuropathy (Fig. 1d-f) consisting of mild dystrophic change with scattered fibers with rimmed vacuoles (Fig. 1e,j-l), as well as group atrophy and fiber type grouping (Fig. 1d,f).

The clinical information and materials from patients were obtained for diagnostic purposes with written informed consent. All experiments in this study were approved by the Ethical Committee of NCNP. In both patients, a possibility of facioscapulohumeral muscular dystrophy 1 was excluded by long range $\mathrm{PCR}^{10}$.

Mutations in known causative genes for myopathy with protein aggregations/rimmed vacuoles were further screened in Patient 1 by an Ion PGM sequencer coupled with an MFM targeted gene panel. Coverage of the $V C P$ locus was $100 \%$ at a depth of 20 reads. We identified a novel missense variant, c.376A $>\mathrm{G}$ (p.Ile126Val), within exon 4 in $V C P$ (Fig. 2a). Follow-up sequencing was carried out and confirmed the variant. p.Ile126Val was located within the NTD, cofactor and ubiquitin binding function (Fig. 2b). The isoleucine residue was highly conserved from humans to yeast, except for in Drosophila melanogaster (Fig. 2c). Six of 10 mutation predictors showed the variant as "Deleterious" or "Disease causing" (Supplementary Table S2).

In Patient 2, the nucleotide sequences of the exons and exon/intron boundaries in VCP were further determined by the Sanger method based upon the clinical phenotype. A novel variant, c.259G $>\mathrm{T}$ (p.Val87Phe), in exon 3 was identified. The primer sequences for mutation screening were $5^{\prime}$-cagggctgctgcttactcc- $3^{\prime}$ (forward primer) and $5^{\prime}$ ctgtaatgcaggctatctctgg- $3^{\prime}$ (reverse). This mutated valine is also located within the NTD (Fig. 2b) and is evolutionally conserved (Fig. 2c). SIFT, PolyPhen-2 and Mutation Taster predicted the mutation to be "Tolerated" with a score of 0.14 , "Possibly damaging" with a score 0.85 and "Disease causing" with a score of 0.99 , respectively.

Finally, neither variant had been deposited in any databases, including dbSNP, 1000 Genomes, Exome Aggregation Consortium, Human Gene Mutation Database, Human Genetic Variation Database, Integrated Japanese Genome Variation Database or ClinVar (as the end of February 2018). Hence, the two variants were considered to be novel.

Cytoplasmic and nuclear inclusions and rimmed vacuoles, which are common pathological changes in VCP-related myopathy, were observed in muscles from our patients (Fig. 1g-l). These inclusions were positive for ubiquitin, TDP-43, SQSTM1 and VCP as previously reported ${ }^{4}$, suggesting again the impairment of ubiquitin-proteasome-degradation systems; consequently, autophagy induction is implicated in the pathomechanism based on the malfunction of mutated VCP in this disease.
In this study, we identified two novel variants, both of which cause amino acid substitutions. The variant in Patient 2, p.Val87Phe, was classified as "Possibly damaging" or "Disease-causing" in two of three predictions, and the residue is well conserved among species, suggesting that it is pathogenic mutation. In contrast, in the variant in Patient 1, the isoleucine of p.Ile126Val was replaced with valine in Drosophila melanogaster, suggesting that the mutation will be a relatively benign variant for pathogenesis. However, regarding variant p.Ile126Val, Patient 1 showed clinical symptoms, muscle pathology and protein aggregate patterns comparable to those in VCP disease. Six of 10 mutation predictors also showed the variant as "Deleterious" or "Disease causing" (Supplementary Table S2). Furthermore, it is to be noted that a different missense substitution at this amino acid residue (p.Ile126Phe) has been reported to be a pathogenic mutation $^{5}$. Consequently, our clinical and in silico data suggest that p.Ile126Val is likely to be a milder mutation involved in the pathogenesis of VCP.

$\mathrm{VCP}$ is a homo-hexameric complex with each monomer comprising the NTD and a pair of ATPase domains (D1 and D2) ${ }^{11}$. As in almost all previous mutations, our substitution sites (Val87, Ile126) were also located in NTD and were conformationally aligned nearby (Fig. 2b,d). Fine structural analyses of full-length VCP or nucleotides in the NTD-D1 domain by crystalography ${ }^{12}$ and NMR spectroscopy ${ }^{13}$ unraveled that the reported missense mutations in NTD had roles in the malfunction of VCP molecules. Based on these analyses, the importance of dynamic structural changes around the Val87 site for disease pathogenesis has been discussed. Further analyses of genetic variations, structural changes and the ATPase activity of mutated VCP and the resulting cellular and muscle phenotypes will improve our understanding of the pathomechanism of VCP diseases and provide insights into the development of therapies.

\section{HGV Database}

The relevant data from this Data Report are hosted at the Human Genome Variation Database at https://doi.org/10.6084/m9.figshare.hgv.2312, https://doi. org/10.6084/m9.figshare.hgv.2315.

\section{Acknowledgements}

We are very grateful to the patient who participated in this study. We also thank the members of the Department of Clinical Development at the Medical Genome Center for their excellent technical assistance. This study was partially supported by an Intramural Research Grant (29-4 to I.N. and Y.K.H.; 28-6 to S. N.) for Neurological and Psychiatric Disorders of NCNP, Research and Development Grants for Comprehensive Research for Persons with Disabilities (17dk0310072h0002 to S.N.), The Integrated Database Construction Program for Clinical Genome Informatics (17kk0205001s0202 to I.N.), the Practical Research Project for Rare/Intractable Diseases (17ek0109285h0001, 16ek0109196h0001 and 17ek0109196h0001 to I.N.), the Program for an Integrated Databases of Clinical and Genomic Information (17kk0205012h0002 to A.I.) from the Japan Agency for Medical Research and Development, AMED, JSPS KAKENHI (JP15H04846 to S.N.; 16 K16597 to S.H.) and the MEXT-Supported Program for the Strategic Research Foundation at Private Universities (5020-03 to Y.K.H.) 


\section{Author details}

'Department of Neuromuscular Research, National Institute of Neuroscience, National Center of Neurology and Psychiatry, Tokyo 187-8551, Japan. ${ }^{2}$ Integrated Graduate School of Medicine, Engineering, and Agricultural Science, University of Yamanashi, Yamanashi 409-3898, Japan. ${ }^{3}$ Department of Clinical Genome Analysis, Medical Genome Center, National Center of Neurology and Psychiatry, Tokyo 187-8551, Japan. ${ }^{4}$ Department of Neurology, National Center Hospital, National Center of Neurology and Psychiatry, Tokyo 187-8551, Japan. ${ }^{5}$ Department of Neurology and Strokology, Nagasaki University Hospital, Nagasaki 852-8501, Japan. ${ }^{6}$ Department of

Pathophysiology, Tokyo Medical University, Tokyo 160-0023, Japan

\section{Conflict of interest}

The authors declare that they have no conflict of interest.

\section{Publisher's note}

Springer Nature remains neutral with regard to jurisdictional claims in published maps and institutional affiliations

Supplementary information is available for this paper at https://doi.org/ 10.1038/s41439-018-0009-7.

Received: 11 March 2018 Revised: 6 April 2018 Accepted: 13 April 2018. Published online: 30 May 2018

\section{References}

1. Meyer, H. \& Weihl, C. C. The VCP/p97 System at a glance: connecting cellular function to disease pathogenesis. J. Cell. Sci. 127, 3877-3883 (2014).

2. Evangelista, T., Weihl, C. C., Kimonis, V. \& Lochmüller, H., VCP related diseases Consortium. 215th ENMC International Workshop VCP-related multi-system proteinopathy (IBMPFD) 13-15 November 2015, Heemskerk, The Netherlands. Neuromuscul. Disord. 26, 535-547 (2016).

3. Johnson, J. O. et al. Exome sequencing reveals VCP mutations as a cause of familial ALS. Neuron 68, 857-864 (2010).

4. Shi, Z. et al. Characterization of the Asian myopathy patients with VCP mutations. Eur. J. Neurol. 19, 501-509 (2012).

5. Matsubara, S. et al. Nuclear inclusions mimicking poly(A)-binding protein nuclear 1 inclusions in a case of inclusion body myopathy associated with Paget disease of bone and frontotemporal dementia with a novel mutation in the valosin-containing protein gene. Neuromuscul. Disord. 26, 436-440 (2016)

6. Taylor, J. P. Multisystem proteinopathy: intersecting genetics in muscle, bone, and brain degeneration. Neurology 85, 658-660 (2015).

7. van den Boom, J. \& Meyer, H. VCP/p97-Mediated Unfolding as a Principle in Protein Homeostasis and Signaling. Mol. Cell. 69, 182-194 (2018).

8. Watts, G. D. et al. Inclusion body myopathy associated with Paget disease of bone and frontotemporal dementia is caused by mutant valosin-containing protein. Nat. Genet. 36, 377-381 (2004).

9. Nishikawa, A., Mitsuhashi, S., Miyata, N. \& Nishino, I. Targeted massively parallel sequencing and histological assessment of skeletal muscles for the molecular diagnosis of inherited muscle disorders. J. Med. Genet. 54, 134-144 (2017).

10. Goto, K., Nishino, I. \& Hayashi, Y. K. Rapid and accurate diagnosis of facioscapulohumeral muscular dystrophy. Neuromuscul. Disord. 16, 256-261 (2006).

11. DeLaBarre, B. \& Brunger, A. T. Complete structure of p97/valosin-containing protein reveals communication between nucleotide domains. Nat. Struct. Biol. 10, 856-863 (2003).

12. Tang, W. K. et al. A novel ATP-dependent conformation in p97 N-D1 fragment revealed by crystal structures of disease-related mutants. EMBO J. 29, 2217-2229 (2010).

13. Schuetz, A. K. \& Kay, L. E. A Dynamic molecular basis for malfunction in disease mutants of p97NCP. elife 5, e20143 (2016). 